\title{
Jaynes-Cummings model with a collective atomic mode
}

\author{
Shi-Biao Zheng \\ Department of Electronic Science and Applied Physics \\ Fuzhou University \\ Fuzhou 350002, P. R. China
}

(Dated: November 28, 2018)

\begin{abstract}
We study the dynamics of a single control atom and an atomic sample interacting with a nonresonant cavity mode. The control atom is driven by an auxiliary classical field. Under certain conditions, the coherent energy exchange between the control atom and the atomic sample induced by the cavity mode is described by the Jaynes-Cummings model. The idea provides a possibility for quantum state engineering and reconstruction for collective atomic modes.
\end{abstract}

PACS numbers: PACS number: 42.50.Pq, 42.50.Fx, 03.67.Mn

The Jaynes-Cummings model (JCM) [1,2], which describes the interaction of a two-level atom and a quantized electromagnetic field, is a cornerstone for treatment of the interaction between light and matter in quantum optics. It gives arise to many quantum phenomena that can not be explained in classical terms, such as the collapses and revivals of the atomic population inversion [3], squeezing of the field [4], and atom-cavity entanglement [5]. Recent experiments with Rydberg atoms and microwave photons in a superconducting cavity have turned the JCM from a theoretical curiosity to a useful and testable enterprise [6]. Such a system is also suitable for quantum state engineering and quantum information processing. Up to now, Fock states [7], Schrodinger cat states [8-10], and entangled states $[11,12]$ have been produced in cavity quantum electrodynamics (QED) experiments. The quantum logic gate using a Rydberg atom and a cavity field as quantum bits has also been experimentally realized [13].

In the past few years, there has been interest in the implementation of the Jaynes-Cummings Hamiltonian with the quantized field replaced by other bosonic systems. A typical example is the laser-assisted coupling between the internal and external degrees of freedom of a trapped ion, in which the excitation (deexcitation) of the electronic state is accompanied by the annihilation (creaction) of a phonon in the vibrational mode [14]. Thanks to the advances in ion trapping and laser cooling, various quantum states and quantum information processsors have been experimentally implemented with trapped ions [15-18]. Recently, Molmer has shown that the dynamics of a pair of Bose-Einstein condensates can be described by the Jaynes-Cummings Hamiltonian [19].

In this paper, we study the dynamics of a system consisting of a single control atom and an N-atom sample, both of which are couple to a common cavity mode. Meanwhile, the control atom is driven by an auxiliary clasiscal field. Through suitable dispersive interactions, we show that the dynamics of the coupled control atom and atomic ensemble can be described by an effective Jaynes-Cummings model, in which the collective ensemble atomic spin is treated as a bosonic mode. One can switch from resonant JCM to nonresonant JCM by tuning the Rabi frequency of the classical field. Through suitable choices of interaction strengths and interaction times, one may in principle generate various quantum states of the collective atomic mode. The idea can be generalized to generate entangled states for two or more atomic samples.

We assume that each atom has one excited state $|e\rangle$ and one ground state $|g\rangle$. The Hamiltonian for the whole system is (assuming $\hbar=1$ )

$$
H=H_{0}+H_{i},
$$

where

$$
\begin{gathered}
H_{0}=\omega_{c} a^{+} a+\omega_{0}\left(S_{z, c}+\sum_{j=1}^{N} S_{z, j}\right), \\
H_{i}=\Omega\left(S_{c}^{-} e^{i \omega_{d} t}+S_{c}^{+} e^{-i \omega_{d} t}\right)+g\left(a^{+} S_{c}^{-}+a S_{c}^{+}\right)+g \sum_{j=1}^{N}\left(a^{+} S_{j}^{-}+a S_{j}^{+}\right) .
\end{gathered}
$$

$S_{z, c}=\frac{1}{2}\left(\left|e_{c}\right\rangle\left\langle e_{c}|-| g_{c}\right\rangle\left\langle g_{c}\right|\right), S_{c}^{+}=\left|e_{c}\right\rangle\left\langle g_{c}\right|$, and $S_{c}^{-}=\left|g_{c}\right\rangle\left\langle e_{c}\right|$ are the inversion, rising, and lowering operators for the control atom, $S_{z, j}, S_{j}^{-}$, and $S_{j}^{+}$denote the inversion, rising, and lowering operators for the jth atom in the atomic sample, $a^{+}$and $a$ are the creation and annihilation operators for the cavity mode, $\omega_{0}$ is the atomic transition 
frequency, $\omega_{c}$ is the cavity frequency, $\omega_{d}$ is the frequency of the classical field, $g$ is the atom-cavity coupling strength, and $\Omega$ is the Rabi frequency of the classical field. Under the condition $\delta_{c}=\omega_{0}-\omega_{c} \gg g \sqrt{N(\bar{n}+1)}$, with $\bar{n}$ being the mean photon number of the cavity field, there is no energy exchange between the atomic system and the cavity. The dispersive atom-cavity interaction leads to photon-number dependent Stark shifts and dipole couplings for the atomic system. In the case $\delta_{d}=\omega_{0}-\omega_{d} \gg \Omega$, the classical field only induces a Stark shift. Then the effective Hamiltonian is $[20]$

$$
\begin{aligned}
H_{e}= & \lambda_{d}\left(\left|e_{c}\right\rangle\left\langle e_{c}|-| g_{c}\right\rangle\left\langle g_{c}\right|\right)+\lambda_{c}\left(\left|e_{c}\right\rangle\left\langle e_{c}\left|a a^{+}-\right| g_{c}\right\rangle\left\langle g_{c}\right| a^{+} a\right) \\
& \left.+\lambda_{c} \sum_{j=1}^{N}\left(\left|e_{j}\right\rangle\left\langle e_{j}\left|a a^{+}-\right| g_{j}\right\rangle\left\langle g_{j}\right| a^{+} a\right)+\lambda_{c} \sum_{j}^{N}\left(S_{c}^{+} S_{j}^{-}+S_{c}^{-} S_{j}^{+}\right)+\sum_{j, k=1}^{N} S_{j}^{+} S_{k}^{-}\right], \\
j \neq & k,
\end{aligned}
$$

where $\lambda_{d}=\Omega^{2} / \delta_{d}$ and $\lambda_{c}=g^{2} / \delta_{c}$.

Since $\left[a^{+} a, H_{e}\right]=0$, the photon-number conserves during the interaction. If the cavity is initially in the vacuum state it will remain in this state and the effective Hamiltonian reduces to

$$
\begin{aligned}
H_{e}= & \lambda_{d}\left(\left|e_{c}\right\rangle\left\langle e_{c}|-| g_{c}\right\rangle\left\langle g_{c}\right|\right)+\lambda_{c}\left|e_{c}\right\rangle\left\langle e_{c}\right| \\
& +\lambda_{c} \sum_{j}^{N}\left(S^{+} S_{j}^{-}+S^{-} S_{j}^{+}\right)+\lambda_{c} \sum_{j, k=1}^{N} S_{j}^{+} S_{k}^{-}, \\
j \neq & k .
\end{aligned}
$$

Setting

$$
\begin{aligned}
b & =\frac{1}{\sqrt{N}} \sum_{j=1}^{N} S_{j}^{-}, \\
b^{+} & =\frac{1}{\sqrt{N}} \sum_{j=1}^{N} S_{j}^{+}, \\
n_{b} & =\sum_{j=1}^{N}\left|e_{j}\right\rangle\left\langle e_{j}\right|,
\end{aligned}
$$

we have

$$
\begin{aligned}
{\left[b, b^{+}\right] } & =1-\frac{2}{N} n_{b}, \\
{\left[n_{b}, b^{+}\right] } & =b^{+} \\
{\left[n_{b}, b\right] } & =-b .
\end{aligned}
$$

Suppose that $N \gg 1, \bar{n}_{b}$, with $\bar{n}_{b}$ being the average excitation number of the atomic sample. Then $b, b^{+}$can be regarded as the bosonic operators and the atomic sample can be regarded as a bosonic system. The Hamiltonian can be rewritten as

$$
\begin{aligned}
H_{e}= & \left(2 \lambda_{d}+\lambda_{c}\right) S_{z, c}+\sqrt{N} \varepsilon b^{+} b \\
& +\varepsilon\left(S_{c}^{+} b+S_{c}^{-} b^{+}\right),
\end{aligned}
$$

where $\varepsilon=\sqrt{N} \lambda_{c}$. Here we have discarded the constant energy $\lambda_{c} / 2$. The Hamiltonian $H_{e}$, showing complete analogy with the Jaynes-Cummings Hamiltonian, describes the oscillatory exchange of an excitation between the control atom and collective atomic mode.

Under the condition $2 \lambda_{d}=(N-1) \lambda_{c}$, the Hamiltonian $H_{e}$, describing the resonant coupling between the control atom and the atomic mode, leads to the transition

$$
\begin{aligned}
\left|e_{c}\right\rangle|n\rangle & \rightarrow e^{-i \sqrt{N} \varepsilon t}\left[\cos (\varepsilon t)\left|e_{c}\right\rangle|n\rangle-i \sin (\varepsilon t)\left|g_{c}\right\rangle|n+1\rangle\right], \\
|g\rangle|n+1\rangle & \rightarrow e^{-i \sqrt{N} \varepsilon t}\left[\cos (\varepsilon t)\left|g_{c}\right\rangle|n+1\rangle-i \sin (\varepsilon t)\left|e_{c}\right\rangle|n\rangle\right],
\end{aligned}
$$


where $|n\rangle$ denotes the Fock state for the atomic mode. The dynamics provides a possibility for realizing various intersting phenomena for the atomic matter wave field. The coupling also makes it possible to engineer particular quantum states of the atomic sample. Suppose that the system is intially in the state $\left|e_{c}\right\rangle|0\rangle$. After an interaction time $t_{1}=\pi / 2 \varepsilon$, the collective atomic mode evolves to the Fock state $|1\rangle$, with the control atom left in the ground state $\left|g_{c}\right\rangle$. We then drive the control atom to the excited state $\left|e_{c}\right\rangle$ via a classical field. After a second interaction time $t_{2}=\pi / 2 \sqrt{2} \varepsilon$, the collective atomic mode evolves to the two-excitation Fock state $|2\rangle$. Repeating the procedure we can generate Fock states for the collective atomic mode with larger excitation-numbers.

The coupling can also be used to generate Schrodinger cat states for the collective atomic mode. Suppose that the control atom is initially in the ground state $\left|g_{c}\right\rangle$ and the atomic sample initially in the coherent state $|\alpha\rangle\left(\alpha=|\alpha| e^{i \theta}\right)$. Under the conditions $|\alpha| \gg 1$ and $g t \ll 4 \bar{n}\left(\bar{n}=|\alpha|^{2}\right)$ the interaction leads to [21]

$$
|\psi(t)\rangle=\frac{1}{\sqrt{2}}\left[e^{-i \sqrt{\bar{n}} \varepsilon t / 2}\left|\alpha^{+}(t)\right\rangle\left|\phi_{a}^{+}(t)\right\rangle-e^{-i \sqrt{\bar{n}} \varepsilon t / 2}\left|\alpha^{+}(t)\right\rangle\left|\phi_{a}^{+}(t)\right\rangle\right],
$$

where

$$
\begin{gathered}
\left|\alpha^{ \pm}(t)\right\rangle=e^{-\bar{n} / 2} \sum_{n} e^{ \pm(n-\bar{n})^{2} \varepsilon t / 8 \bar{n}^{-3 / 2}} \frac{\left(\alpha e^{-i \sqrt{N} \varepsilon t} e^{\mp i \varepsilon t / 2 \sqrt{\bar{n}}}\right)^{n}}{\sqrt{n !}}, \\
\left|\phi_{c}^{ \pm}(t)\right\rangle=\frac{1}{\sqrt{2}}\left(e^{i \theta} e^{\mp i \varepsilon t / 2 \sqrt{\bar{n}}}\left|e_{c}\right\rangle \pm\left|g_{c}\right\rangle\right) .
\end{gathered}
$$

Thus the collective atomic mode splits into two quasicoherent components with different phases correlated with the states of the control atom. As shown in the Jaynes-Cummings dynamics with a cavity field [22], the mesoscopic coherence and decoherence of the collective atomic mode can be revealed by the collapses and revivals of the Rabi oscillations. We can reconstruct the quantum states of the collective atomic mode by detecting the state of the control atom after the resonant Jaynes-Cummings dynamics [23,24].

Set $\delta=2 \lambda_{d}-(N-1) \lambda_{c} \gg \varepsilon$. Then the probability that the control atom exchanges energy with the atomic sample is negligible and the effective Hamiltonian reduces to [8]

$$
H_{e}=\frac{\varepsilon^{2}}{\delta}\left(\left|e_{c}\right\rangle\left\langle e_{c}\left|b b^{+}-\right| e_{c}\right\rangle\left\langle e_{c}\right| b^{+} b\right) \text {. }
$$

Suppose that the atom is initially in the superposition state $\frac{1}{\sqrt{2}}\left(\left|e_{c}\right\rangle+\left|g_{c}\right\rangle\right)$ and the atomic sample initially in the coherent state $|\alpha\rangle$. The effective Hamiltonian gives arise to the Schrödinger cat state

$$
|\psi(t)\rangle=\frac{1}{\sqrt{2}}\left[e^{-i \varepsilon^{2} t / \delta}\left|\alpha e^{-i \varepsilon^{2} t / \delta}\right\rangle\left|e_{c}\right\rangle+\left|\alpha e^{i \varepsilon^{2} t / \delta}\right\rangle\left|g_{c}\right\rangle\right]
$$

The effective Hamiltonian can also be used to directly measure the Wigner function of the atomic matter wave field $[25]$.

The idea can be used to generate entangled states for two atomic samples. Suppose that a control atom and two atomic samples dispersively interact with the vacuum cavity. Meanwhile, the control atom is driven by a nonresonant classical field. Under the above mentioned conditions, the effective Hamiltonian is

$$
\begin{gathered}
H_{e}=\left(2 \lambda_{d}+\lambda_{c}\right) S_{z, c}+\sqrt{N} \varepsilon\left(b_{1}^{+} b_{1}+b_{2}^{+} b_{2}+b_{1}^{+} b_{2}+b_{2}^{+} b_{1}\right) \\
+\varepsilon\left[S_{c}^{+}\left(b_{1}+b_{2}\right)+S_{c}^{-}\left(b_{1}^{+}+b_{2}^{+}\right)\right],
\end{gathered}
$$

where $b_{1}$ and $b_{2}$ represent the annihilation operators for the two collective atomic modes, respectively. Suppose that the control atom is initially in the excited state $\left|e_{c}\right\rangle$ and the two atomic samples initially in the vacuum state $\left|0_{1}\right\rangle\left|0_{2}\right\rangle$. In the case that $2 \lambda_{d}=(2 N-1) \lambda_{c}$, the evolution of the system is

$$
|\psi(t)\rangle=e^{-i 2 \sqrt{N} \varepsilon t}\left[\cos (\sqrt{2} \varepsilon t)\left|e_{c}\right\rangle\left|0_{1}\right\rangle\left|0_{2}\right\rangle-i \sin (\sqrt{2} \varepsilon t)\left|g_{c}\right\rangle\left(\left|1_{0}\right\rangle\left|0_{2}\right\rangle+\left|0_{0}\right\rangle\left|1_{2}\right\rangle\right) / \sqrt{2}\right]
$$

After an interaction time $t=\pi / 2 \sqrt{2} \varepsilon$, the two collective atomic modes evolves to the maximally entangled state

$$
\left|\psi_{m}\right\rangle=e^{-i \sqrt{N} \pi / \sqrt{2}}\left(\left|1_{0}\right\rangle\left|0_{2}\right\rangle+\left|0_{0}\right\rangle\left|1_{2}\right\rangle\right) / \sqrt{2}
$$


with the control atom left in the ground state $\left|g_{c}\right\rangle$.

We note that the entanglement operation can be applied to the system involving $\mathrm{n}$ atomic samples. In this case, the effective Hamiltonian is

$$
\begin{gathered}
H_{e}=\left(2 \lambda_{d}+\lambda_{c}\right) S_{z, c}+\sqrt{N} \varepsilon \sum_{j=1}^{n} \sum_{k=1}^{n} b_{j}^{+} b_{k} \\
+\varepsilon\left(S_{c}^{+} \sum_{j=1}^{n} b_{j}+S_{c}^{-} \sum_{k=1}^{n} b_{j}^{+}\right) .
\end{gathered}
$$

We again assume that the control atom is initially in the excited state $\left|e_{c}\right\rangle$ and the atomic samples initially in the state $\left|0_{1}\right\rangle\left|0_{2}\right\rangle \ldots\left|0_{n}\right\rangle$. With the choice $2 \lambda_{d}=(n N-1) \lambda_{c}$, we obtain the state evolution

$$
\begin{aligned}
|\psi(t)\rangle= & e^{-i n \sqrt{N} \varepsilon t}\left[\cos (\sqrt{n} \varepsilon t)\left|e_{c}\right\rangle\left|0_{1}\right\rangle\left|0_{2}\right\rangle \ldots\left|0_{n}\right\rangle\right. \\
& \left.-i \sin (\sqrt{n} \varepsilon t)\left|g_{c}\right\rangle\left(\left|1_{0}\right\rangle\left|0_{2}\right\rangle \ldots\left|0_{n}\right\rangle+\left|0_{0}\right\rangle\left|1_{2}\right\rangle\left|0_{3}\right\rangle \ldots\left|0_{n}\right\rangle+\ldots+\left|0_{0}\right\rangle\left|0_{2}\right\rangle \ldots\left|0_{3}\right\rangle\left|1_{n}\right\rangle\right) / \sqrt{n}\right] .
\end{aligned}
$$

After an interaction time $t=\pi / 2 \sqrt{n} \varepsilon$, the atomic modes evolves to

$$
\left|\psi_{m}(t)\right\rangle=e^{-i \sqrt{n N} \pi / 2}\left(\left|1_{0}\right\rangle\left|0_{2}\right\rangle \ldots\left|0_{n}\right\rangle+\left|0_{0}\right\rangle\left|1_{2}\right\rangle\left|0_{3}\right\rangle \ldots\left|0_{n}\right\rangle+\ldots+\left|0_{0}\right\rangle\left|0_{2}\right\rangle \ldots\left|0_{3}\right\rangle\left|1_{n}\right\rangle\right) / \sqrt{n}
$$

This is a $\mathrm{W}$ state [26], whose entanglement is robust against qubit loss, global dephasing, and qubit flip noise. Due to the robustness multiqubit W states might lead to stronger nonclassicality [27] than the Greeberger-Horne-Zeilinger states [28] and be useful in quantum information processing [29].

It is necessary to address the experimental implementation of the proposed model. In recent cavity QED experiments [30,31], Cs atoms were trapped in an optical cavity, and the $6 S_{1 / 2}, F=4 \rightarrow 6 P_{3 / 2}, F=4$ transition was coupled to the cavity mode. The corresponding coupling strength is $g=2 \pi \times 34 M H z$. The decay rates for the atomic excited state and the cavity mode are $\Gamma=2 \pi \times 2.6 \mathrm{M} \mathrm{Hz}$ and $\kappa=2 \pi \times 4.1 \mathrm{MHz}$, respectively. The decoherence rate of the atomic sample due to atomic spontaneous emission is given by the single-atom spontaneous emission rate [32]. In order to suppress the influence of the atomic spontaneous emission one should use Raman coupling. Suppose that two ground states are coupled to the excited state through the cavity mode of coupling $g$ and a classical field of coupling $\alpha$. The cavity mode and classical field are detuned from the respective transitions by the amounts $\Delta+\delta$ and $\Delta$, respectively. Under the condition $\Delta \gg g, \alpha, \delta$ the excited state can be adiabatically eliminated and the two ground states are coupled to the cavity mode through Raman process. The Raman coupling strength is $g^{\prime}=\frac{g \alpha}{2}\left(\frac{1}{\Delta+\delta}+\frac{1}{\Delta}\right)$ [33]. Suppose that $\delta \gg g^{\prime}$. Then the Raman coupling is far off-resonant and the cavity mode is virtually excited. This leads to the coupling between the control atom and the atomic sample. The entire process is a four-photon transition [34]. Meanwhile, the energy difference between the two ground states of the control atom can be modified through an additional classical field. Set $N=10^{4}, \Delta=100 \mathrm{~g}, \delta=10 \mathrm{~g}$, and $\alpha=g$. Then the coupling strength between the control atom and the collective atomic mode is $\varepsilon=\sqrt{N} g^{\prime 2} / \delta \simeq 2 \pi \times 3.1 \times 10^{4} \mathrm{~Hz}$. Both the atomic system and the cavity mode are virtually excited. The effective decoherence rates due to the atomic spontaneous emission and cavity decay are $\Gamma^{\prime}=\Gamma g^{2} / \Delta^{2}=2 \pi \times 260 H z$ and $\kappa^{\prime}=\kappa g^{\prime 2} / \delta^{2} \simeq 2 \pi \times 3.7 H z$, respectively. In this case both the effective decoherence rates $\Gamma^{\prime}$ and $\kappa^{\prime}$ are much smaller than the coupling strength $\varepsilon$. For the generation of the Fock state $|1\rangle$, the required interaction time is $t_{1}=\pi / 2 \varepsilon \simeq 8.1 \mu \mathrm{s}$. The infidelity induced by the decoherence is on the order of $\left(\Gamma^{\prime}+\kappa^{\prime}\right) t_{1} \simeq 1.3 \times 10^{-2}$.

It should be noted that the derived Jaynes-Cummmings dynamics is valid in the Lamb-Dicke regime. Recently, a single ${ }^{40} \mathrm{Ca}^{+}$ions was localized at a fixed position in the cavity with a high precision for a long time [35]. In a more recent experiment [36], the localization to the Lamb-dicke limit of the axial motion was demonstrated for a single Cs atom trapped in an optical cavity. In order to couple only the control atom to the additional classical field without coupling the $\mathrm{N}$-atom sample as well one should put the atomic sample in a lattice-like structure and address selectively the control atom by a focused external beam.

In conclusion, we have shown how to realize the JCM with the collective atomic bosonic mode. The coupling between the control atom and the atomic sample is induced by the nonresonant cavity mode, which is always in the vacuum state. Thus the evolution of the system is insensitive to cavity decay. The dynamics provides a possibility for realizing and reconstructing various quantum states of matter wave oscillators. The idea can also be used for preparation of entangled states for two atomic samples trapped in two separate cavities. Suppose that each cavity involves a control atom and an atomic sample. In each cavity the control atom and collective atomic mode are first entangled via the four-photon transition. Then we switch off the classical fields which induces the four-photon transition. Meanwhile, we apply an appropriate classical field to the control atom so that it undergoes a resonant 
Raman transition and can emit a photon. Various entangled states for the two collective atomic modes can be produced by detecting photons leaking out of the cavities [37]. This may allows one to test Bell's inequalities [38] with two entangled macroscopic objects. Due to the long coherence lifetime, the matter wave oscillator is suitable for the storage of quantum information. The entanglement between two distant atomic samples has application in quantum communication [32]. Two atomic samples located in separate cavities can also be entangled by using a fiber to connect the cavities [39]. The two control atoms are first entangled via the exchange of an excitation through the fiber. Then the coupling between the control atom and atomic sample in each cavity leads to the entanglement between the two atomic samples. The idea can be generalized to entangle more distant atomic samples.

This work was supported by the National Natural Science Foundation of China under Grant No. 10674025 and the Doctoral Foundation of the Ministry of Education of China under Grant No. 20070386002.

[1] E. T. Jaynes and F. W. Cummings, Proc. IEEE 51, 89 (1963).

[2] B. W. Shore and P. L. Knight, J. Mod. Opt. 40, 1195 (1993).

[3] J. H. Eberly, N. B. Narozhny, and J. J. Sanchez-Mondragon, Phys. Rev. Lett. 44, 1323 (1980).

[4] H. I. Yoo and J. H. Eberly, Phys. Rep. 118, 239 (1985).

[5] S.J.D. Phoenix and P. L. Knight, Ann. Phys. (N.Y.) 186, 381 (1988); S.J.D. Phoenix and P. L. Knight, Phys. Rev. A 44, 6023 (1991).

[6] J. M. Raimond, M. Brune, and S. Haroche, Rev. Mod. Phys. 73, 565 (2001).

[7] P. Bertet et al., Phys. Rev. Lett. 88, 143601 (2002).

[8] M. Brune et al., Phys. Rev. Lett. 77, 4887 (1996).

[9] A. Auffeves et al., Phys. Rev. Lett. 91, 230405 (2003).

[10] T. Meunier et al., Phys. Rev. Lett. 94, 010401 (2005).

[11] S. Osnaghi et al., Phys. Rev. Lett. 87, 037902 (2001).

[12] A. Rauschenbeutel et al., Science 288, 2024 (2000).

[13] A. Rauschenbeutel et al., Phys. Rev. Lett. 83, 5166 (1999).

[14] W. Vogel and R. L. de Matos Filho, Phys. Rev. A 52, 4214 (1995).

[15] D.M.Meekhof et al., Phys. Rev. Lett. 76, 1796 (1996).

[16] D. Leibfried et al., Nature 438, 639 (2005).

[17] H. Häffner et al., Nature 438, 643 (2005).

[18] F. Schmidt-Kaler et al., Nature 422, 408 (2003).

[19] K. Mölmer, cond-mat/0211542 (2002).

[20] S. B. Zheng and G. C. Guo, Phys. Rev. Lett. 85, 2392 (2000).

[21] J. Gea-Banaclothe, Phys. Rev. Lett. 65, 3385 (1990); J. Gea-Banaclothe, Phys. Rev. A 44, 5913 (1991).

[22] G. Morigi, E. Solano, B.-G. Englert, and H. Walther, Phys. Rev. A 65, 040102 (2002).

[23] P. J. Bardroff, E. Mayr and W. P. Schleich, Phys. Rev. A 51 (1995) 4963.

[24] M. S. Kim et al., Phys. Rev. A 58 (1998) R65.

[25] L.G.Lutterbach and L.Davidovich, Phys. Rev. Lett. 78 (1997) 2547.

[26] W. Dür, G. Vidal, and J. I. Cirac, Phys. Rev. A 62, 062314 (2000).

[27] A. Sen(De) et al., Phys. Rev. A 68, 062306 (2003).

[28] D. M. Greenberger, M. A. Horne, and A. Zeilinger, in Bell,s Theorem, Quantum Theory, and Conceptions of the Universe, edited by M.Kafatos (Kluwer, Dordrecht, 1989); D. M. Greenberger, M. A. Horne, A. Shimony, and A. Zeilinger, Am. J. Phys. 58, 1131 (1990).

[29] D. Dru $\beta$ et al., Phys. Rev. A 57, 2368 (1998); J. Joo et al., New J. Phys. 5, 136 (2003).

[30] J. Mckeever et al., quant-ph/0403121

[31] A. Boca et al., quant-ph/0410164.

[32] L. M. Duan et al., Nature 414, 413 (2001).

[33] A. Imamoglu et al., Phys. Rev. Lett. 83, 4204 (1999).

[34] C. A. Sackett et al., Nature 404, 256 (2000).

[35] M. Keller et al., New J. Phys. 6, 95 (2004).

[36] A. D. Boozer et al., quant-ph/0606104.

[37] S. Bose et al., Phys. Rev. Lett. 83, 5158 (1999).

[38] J. S. Bell, Physics 1, 195 (1965).

[39] A. Serafini, S. Mancini, and S. Bose, Phys. Rev. Lett. 96, 010503 (2006). 